\title{
Properties of Polyhexamethylenediamine/Graphene Oxide Composite Fiber Membranes Prepared by Electrospinning
}

\author{
Chiu Wei Ming, ${ }^{1 *}$ Chan Guan $\mathrm{Ru},{ }^{2}$ and Chan Guan Liang ${ }^{2}$ \\ ${ }^{1}$ Ph.D. Program, Graduate Institute of Precision Manufacturing, National Chin-Yi University of Technology, \\ No. 57, Sec. 2, Zhongshan Rd., Taiping Dist., Taichung 41170, Taiwan (R.O.C.) \\ ${ }^{2}$ Department of Chemical and Materials Engineering, National Chin-Yi University of Technology, \\ No. 57, Sec. 2, Zhongshan Rd., Taiping Dist., Taichung 41170, Taiwan (R.O.C.)
}

(Received February 6, 2019; accepted February 6, 2020)

Keywords: graphene oxide, nylon 6,6, electrospinning, air filtration

In this study, an electrospinning solution was first varied, then the ratio of different solvents was varied. The effects of the viscosity and conductivity of the electrospinning solution on the fiber were further investigated to find optimal parameters. It was found that a high viscosity not only increased the fiber diameter, but also sometimes caused agglomeration. A material tester was used to evaluate the mechanical strength of the fiber membrane for different solvent ratios. The optimal solvent ratio was obtained by comparing the effects of different weight ratios of graphene oxide (GO) on the fiber membrane. GO increased the conductivity, and fine fibers were obtained when $0.8 \mathrm{wt} \% \mathrm{GO}$ was added. After adjusting the main parameters of electrospinning, the best fiber film was prepared. By adding a suitable amount of GO, the mechanical strength and thermal cracking properties of the fiber membrane were improved, and the filtration rate of PM2.5 was as high as $98 \%$.

\section{Introduction}

In recent years, electrospinning has become one of the most important methods of preparing nanofibers. This technology can be traced back to the equipment used by Charles and coworkers 1887 with "small plates connected to a motor", and fibers of shellac, beeswax, sealing wax, gutta-percha, and other substances have been obtained. ${ }^{(1)}$ Cooley $^{(2,3)}$ in 1900 and Morton $^{(4)}$ in 1902 obtained patents for electrospinning. Since then, many scientists have also proposed electrospinning technologies and obtained patents.

Electrospinning has become one of the main methods used to effectively prepare nanofiber materials because of its simple manufacturing equipment, low spinning cost, wide variety of spinnable materials, and controllable process. The fibers produced by traditional methods, such as melt spinning and dry/wet spinning, can only reach micron size, but electrospinning can produce nanometer-scale fibers upon tuning the parameters. If a functional powder is also used in the preparation, not only can the physical properties of the fibers be improved, but also the fibers produced can have various functionalities, increasing the diversity of fibers obtained by electrospinning. 
The above applications indicate the high potential of electrospinning for functional applications. The aim of this study was to explore process parameters and combine electrospinning with the fabrication of membranes. The membranes were characterized by their filtration efficiency and demonstrated favorable properties to be explored further for applications in the air filtration sector.

\section{Materials and Methods}

\subsection{Materials}

Graphite, sulfuric acid $\left(\mathrm{H}_{2} \mathrm{SO}_{4}, 95-98 \%\right)$, potassium permanganate $\left(\mathrm{KMNO}_{4}, 98 \%\right)$, polyhexamethylene adipamide, ethanol, acetone, trifluoroacetic acid (TFA), formic acid, and acetic acid were purchased from ECHO CHEMICAL Co., Ltd. All chemicals were of analytical grade and used without further purification.

\subsection{Preparation of graphene oxide (GO)}

GO was obtained by oxidizing graphene via a simplified Hummer's method, which began with mixing graphite and $\mathrm{KMnO}_{4}$ while slowly adding concentrated $\mathrm{H}_{2} \mathrm{SO}_{4}$ in an ice bath and stirring for 5 days until the mixture became a brown paste. Then, $700 \mathrm{ml}$ of $5 \% \mathrm{H}_{2} \mathrm{SO}_{4}$ aqueous solution was added, followed by stirring for $2 \mathrm{~h}$, then $20 \mathrm{ml}$ of $30 \% \mathrm{H}_{2} \mathrm{O}_{2}$ aqueous solution was added to terminate the reaction. The mixture was centrifuged and washed several times with $\mathrm{H}_{2} \mathrm{SO}_{4} / \mathrm{H}_{2} \mathrm{O}_{2}$ aqueous solution to remove excess metal ions, then washed with deionized water to neutralize it. Finally, the precipitate was freeze-dried to obtain GO.

\subsection{Preparation of composite fiber membrane}

Nylon 6,6 plastic was dissolved in TFA/acetone solution. 22 wt\% Nylon 6,6 and TFA/acetone with ratios of 1:1, 3:2, 3:1, and 4:1 and $\mathrm{HCOOH} / \mathrm{CH}_{3} \mathrm{COOH}$ with ratios of 1:1 were used to prepare electrospinning solutions with different ratios. Each solution was mixed with a magnetic stirrer for 1 day at $25{ }^{\circ} \mathrm{C}$ to ensure that the solution completely dissolved, then 0.2 to $2.0 \mathrm{wt} \% \mathrm{GO}$ was added. Before electrospinning, the solution was subjected to ultrasonication for $1 \mathrm{~h}$ to completely disperse $\mathrm{GO}$ in the electrospinning solution. The electrospinning conditions were as follows: (1) The syringe pump flow rate was $0.002 \mathrm{ml} / \mathrm{min}$. (2) The distance between the needle and the collecting plate was $17 \mathrm{~cm}$. (3) The voltage was $19500 \mathrm{~V}$ and the current was $750 \mathrm{~mA}$. (4) The syringe used to prepare the Nylon 6,6 polymer film had a volume of $5 \mathrm{ml}$. (5) The electrospun fibers were collected on a collection plate.

\subsection{Characterization}

The morphologies of the surfaces of GO and the fiber membrane were analyzed by fieldemission scanning electron microscopy (FE-SEM, JSM-6360) with an acceleration voltage 
of $5 \mathrm{kV}$. Transmission electron microscopy (TEM, HITACHI H-7500) was used to observe the internal structure of GO, and a Raman spectrometer (JOBIN-YVON T64000 Micro-PL) was used to probe the characteristic peaks of GO. Fourier transform infrared spectroscopy (FT-IR, Affinity-1) was used to detect the filtered functional groups of the pollutants. A thermogravimetric analyzer (TGA TA-Q500) was used to obtain the decomposition temperature of the composite fiber membrane. The mechanical strength of the membrane was obtained using a universal testing machine (Tinius Olsen, H5KS). The filtration efficiency was evaluated using a handheld microparticle counter (TSI AeroTrak9303).

\section{Results and Discussion}

\subsection{Observation of GO surface morphology by SEM}

It can be seen in Fig. 1(a) that GO was not given sufficient time for oxygenation after ultrasonic oscillation, meaning that the oxygen groups could not effectively be inserted into the intercalation layer, causing the aggregation of GO. Figure 1(b) shows GO after a longer oxygenation time after ultrasonic oscillation.

\subsection{TEM analysis of GO}

TEM was used to observe the GO layer structure and the number of layers, where the latter was determined from the bright and dark parts of the image. The multilayer GO with 4 to 10 layers prepared in this study is shown in Fig. 2(a), and Fig. 2(b) shows a single GO layer.

\subsection{Raman analysis of GO}

We can find the number of defects of GO by calculating the ID/IG value from the Raman spectra. The result is shown in Fig. 3. The ID/IG value of GO prepared in this study was larger

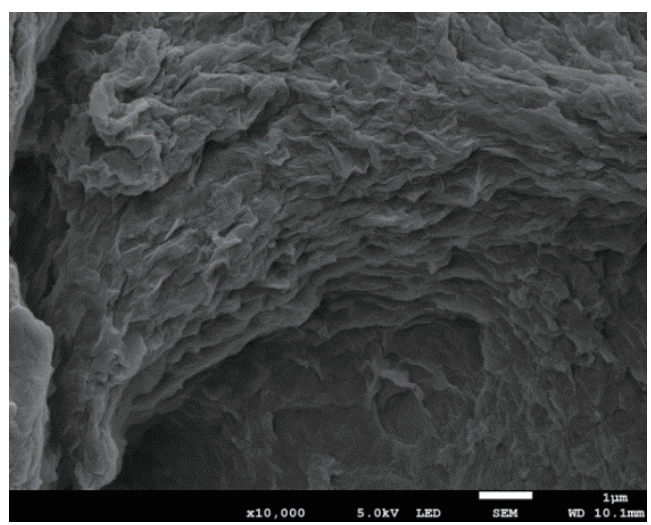

(a)

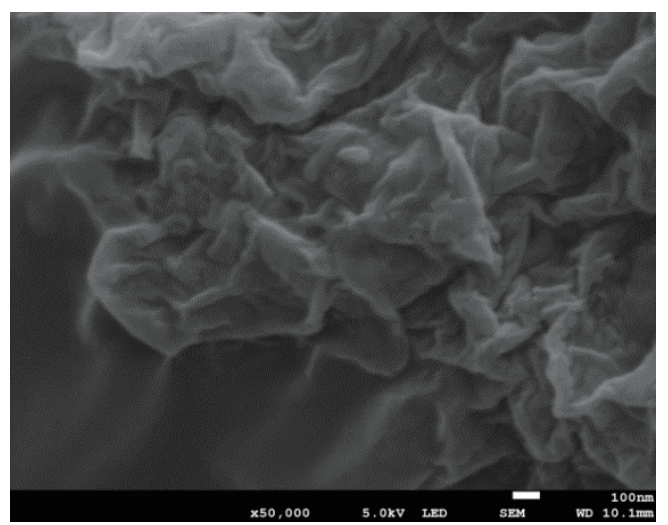

(b)

Fig. 1. SEM images of GO after ultrasonic oscillation (a) with insufficient oxygenation time and (b) after a longer oxygenation time. 


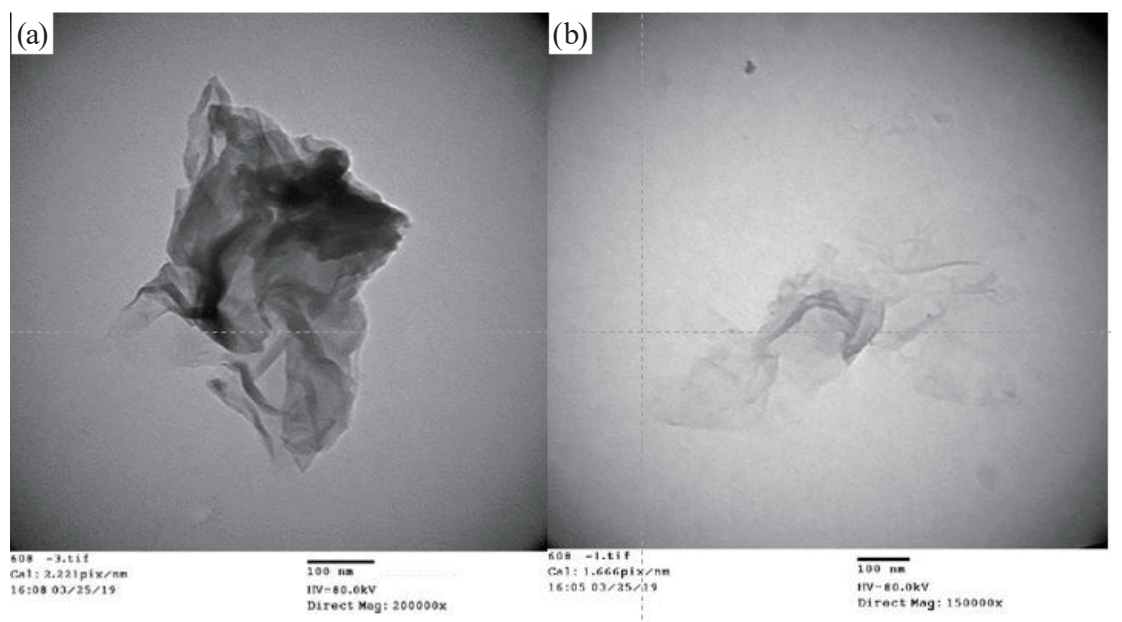

Fig. 2. TEM images of (a) multilayer GO with 4 to 10 layers and (b) single-layer GO.

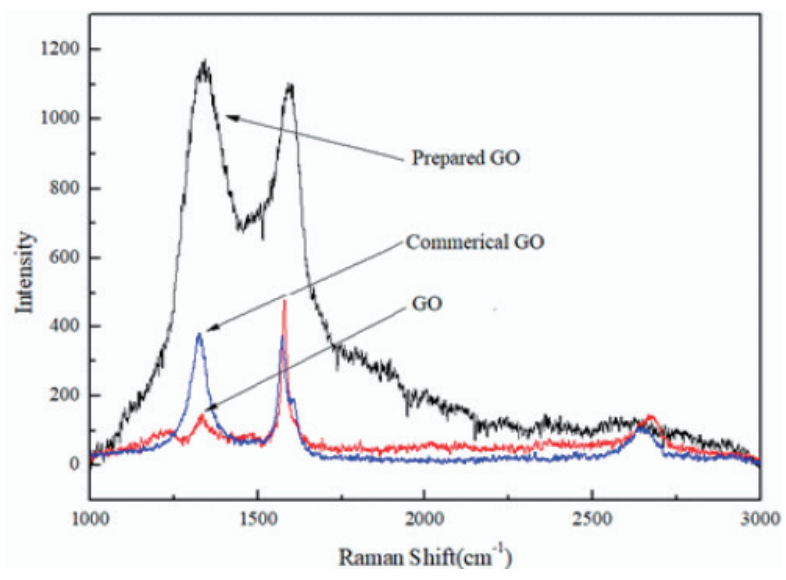

Fig. 3. (Color online) Raman spectra of GO.

than that of commercial GO. This indicates that the material surface structure was destroyed and the number of defects increased. Because of chemical intercalation, the acid solution and strong oxidant create defects and oxygen groups on the graphite surface, then the oxygen groups combine with carbon to form carbon dioxide. Thus, increasing the number of graphene edges increases the D-band value, and the $\mathrm{G}$ band shifts to the right and the 2D band shifts to the left. From this phenomenon, it was found that fewer than 10 graphene layers were prepared. ${ }^{(5)}$

\subsection{Determination of optimum solvent ratio}

The mechanical properties of a fiber membrane are related to the solvents used, so solvent selection is important in this study. Figure 4 shows the tensile stress diagram of the fiber membranes prepared with the above solvent ratios and Table 1 shows the tensile data of the fiber membranes prepared with different solvent ratios. ${ }^{(6)}$ During melting, hydrogen bonds are formed between polymer chains; thus, increasing the amount of TFA increased the stress. The stress was highest for the TFA:acetone ratio of 3:2 and lowest for the ratio of 4:1. It is known 


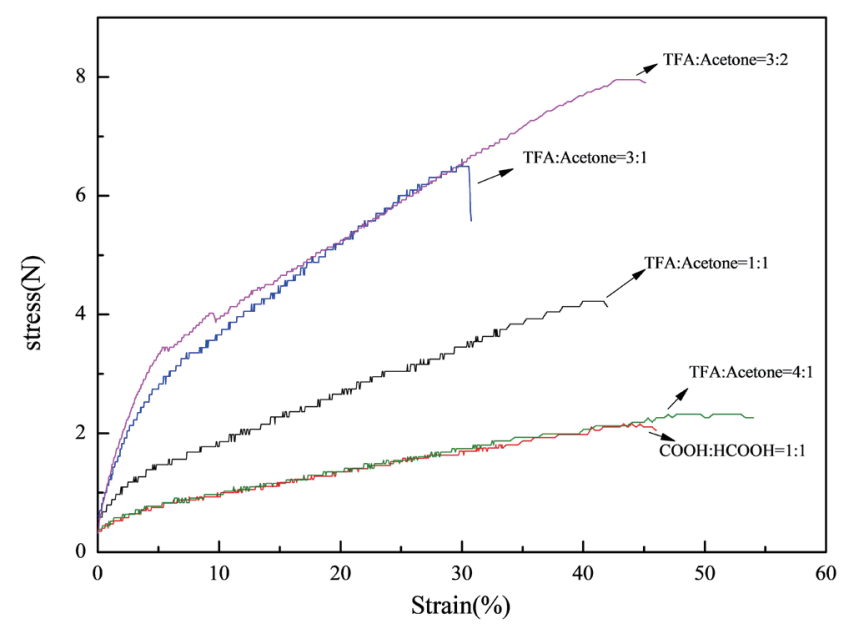

Fig. 4. (Color online) Tensile stress diagram of fiber membranes prepared with different solvent ratios.

Table 1

Tensile data of fiber membranes prepared with different solvent ratios.

\begin{tabular}{lccc}
\hline Sample & Elongation (\%) & Strain (MPa) & Film thickness (mm) \\
\hline Nylon 6,6/TFA:Acetone (1:1) & 42 & 4.22 & 0.0033 \\
Nylon 6,6/Formic acid:Acetic acid (1:1) & 46 & 2.16 & 0.05 \\
Nylon 6,6/TFA:Acetone (3:1) & 30.5 & 6.49 & 0.0033 \\
Nylon 6,6/TFA:Acetone (3:2) & 45.1 & 7.95 & 0.0033 \\
Nylon 6,6/TFA:Acetone (4:1) & 54 & 2.32 & 0.0 \\
\hline
\end{tabular}

from the literature that excessive TFA enhances the degradation of Nylon 6,6 polymer chains, thus decreasing the intermolecular force and the mechanical strength. Therefore, from the SEM images and tensile stress diagram, a TFA:acetone ratio of 3:2 is the optimum ratio for this experiment.

\subsection{Quantitative effect of GO on polymer electrospinning solution}

\subsubsection{Fiber morphology and mechanical properties}

During electrospinning, GO was wrapped in Nylon 6,6. Thus, the injection of GO into Nylon 6,6 can enhance the mechanical properties and heat resistance of the fibers. Moreover, adding GO to the polymer solution can increase conductivity. At this time, the solution has a degradation effect owing to the addition of GO. This caused the fiber size to change and fine fibers to be generated, which are advantageous for air filtration. Furthermore, adding GO can change the viscosity and conductivity of the fibers, which contributes to the formation of a Taylor cone during preparation. As shown in Figs. $5(\mathrm{a})-5(\mathrm{~g})$, increasing the amount of GO increases the viscosity and conductivity, but the change in fiber size is insignificant. When the amount of GO was increased to $0.8 \mathrm{wt} \%$, thin fibers appeared, which affected the tensile strength. The result is shown in Fig. 6. This caused the viscous polymer solution to be extruded by the spinneret during electrospinning. A polymer jet was formed by the electrostatic force 


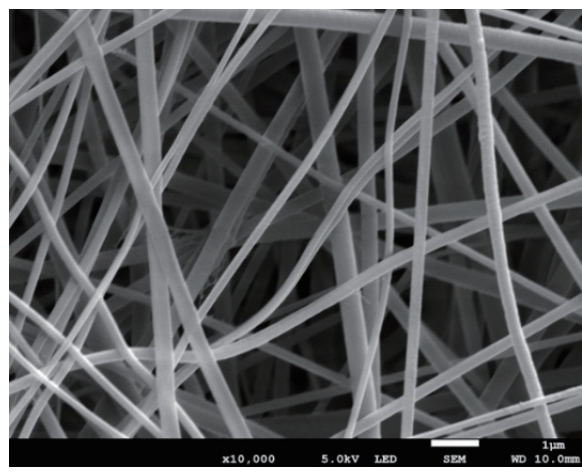

(a)

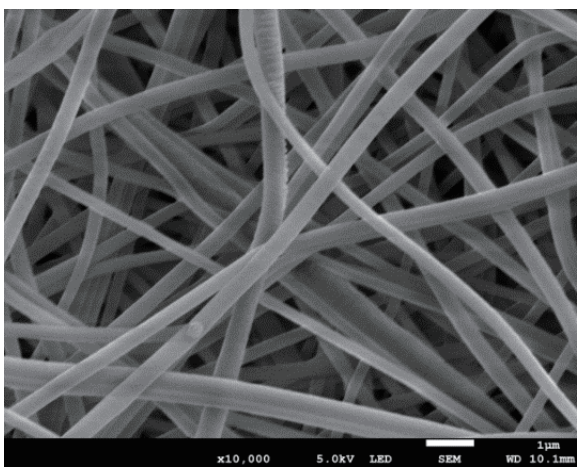

(c)

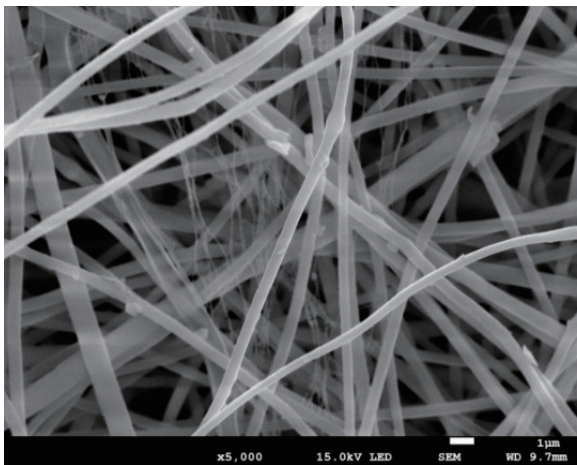

(e)

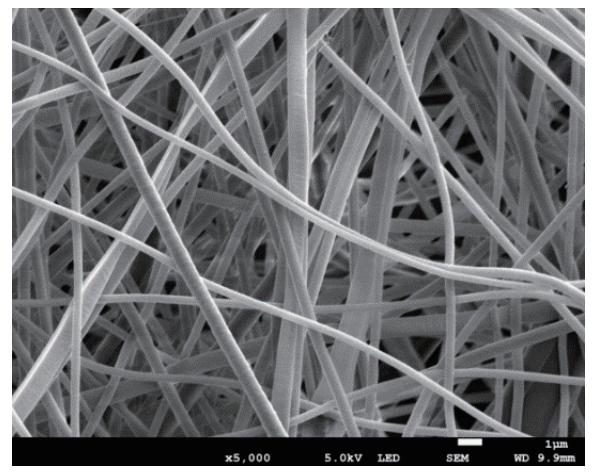

(b)

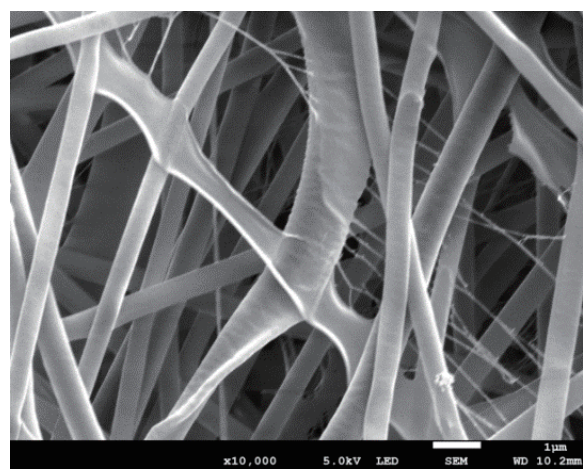

(d)

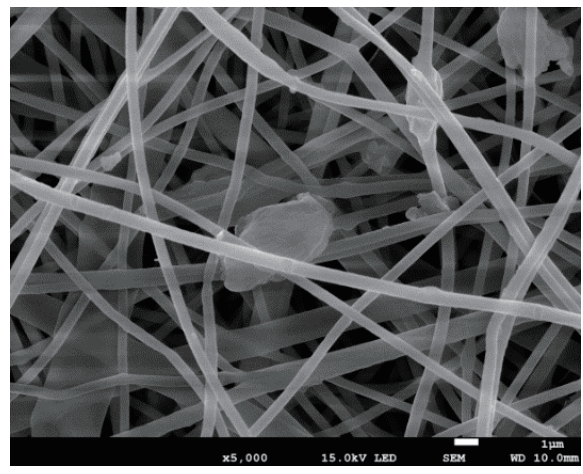

(f)

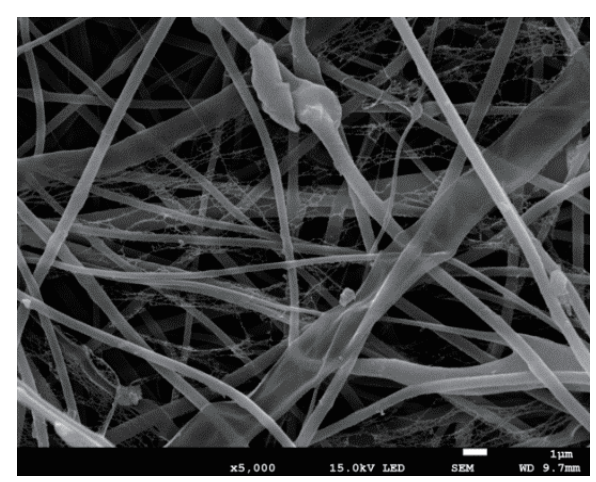

(g)

Fig. 5. SEM images of (a) neat nylon fibers and fibers with (b) 0.2 , (c) 0.5 , (d) 0.8 , (e) 1.0, (f) 1.5, and (g) 2.0 wt $\%$ GO. 
during electrospinning. When the polymer jet was separated from the electrode, the polymer jet had a residual charge, and individual jets started to form thin fibers through cross-linking. ${ }^{(7)}$ In addition to the electrical conductivity and viscosity, the main reason for the formation of thin fibers in this study was that when the polymer solution was stirred, the GO hydroxyl (-OH) and Nylon 6,6 amide group (CO-NH) underwent a cross-linking reaction to form thin fibers. Although increasing the amount of GO can stabilize the structure, when $1.0 \mathrm{wt} \% \mathrm{GO}$ was added, not only did it lead to its agglomeration but also spherical and spindle-shaped beads were observed on the fibers, reducing their quality. When spherical and spindle-shaped beads appear on fibers, the stress will concentrate, adversely affecting their mechanical properties (Table 2).

\subsubsection{Thermal properties}

Different amounts of GO were added to the Nylon 6,6 fiber membranes, and a thermogravimetric analyzer was used to analyze their thermal properties. The result (Table 3) indicated that $T_{d}$ for neat Nylon 6,6 is $352.89{ }^{\circ} \mathrm{C}$ and that $T_{d}$ increased with the amount of GO added, reaching $394.1^{\circ} \mathrm{C}$ for $2.0 \mathrm{wt} \% \mathrm{GO}$, which shows an increase of $41.21^{\circ} \mathrm{C}$. Figure 7 shows the results of the TGA analysis of composite fiber membranes with different GO contents, and

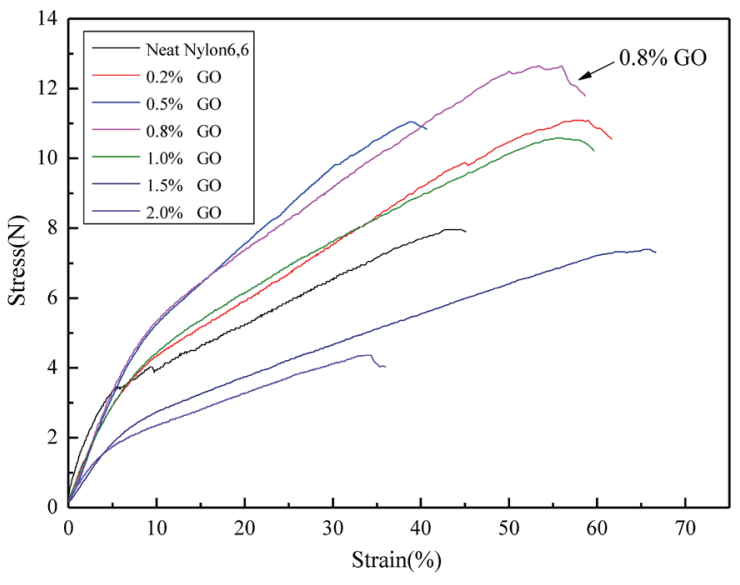

Fig. 6. (Color online) Tensile stress diagram of fiber membranes with different GO contents.

Table 2

Mechanical properties of composite membranes.

\begin{tabular}{lcrc}
\hline Sample & $\begin{array}{c}\text { Strain } \\
(\%)\end{array}$ & $\begin{array}{c}\text { Stress } \\
(\mathrm{MPa})\end{array}$ & $\begin{array}{c}\text { Thickness } \\
(\mathrm{mm})\end{array}$ \\
\hline Neat Nylon 6,6 & 45.1 & 7.95 & 0.0033 \\
$0.2 \%$ GO & 61.6 & 11.09 & 0.135 \\
$0.5 \%$ GO & 40.7 & 11.05 & 0.25 \\
$0.8 \%$ GO & 58.7 & 12.65 & 0.13 \\
$1.0 \%$ GO & 59.7 & 10.59 & 0.177 \\
$1.5 \%$ GO & 66.7 & 7.39 & 0.245 \\
$2.0 \%$ GO & 36 & 4.36 & 0.176 \\
\hline
\end{tabular}

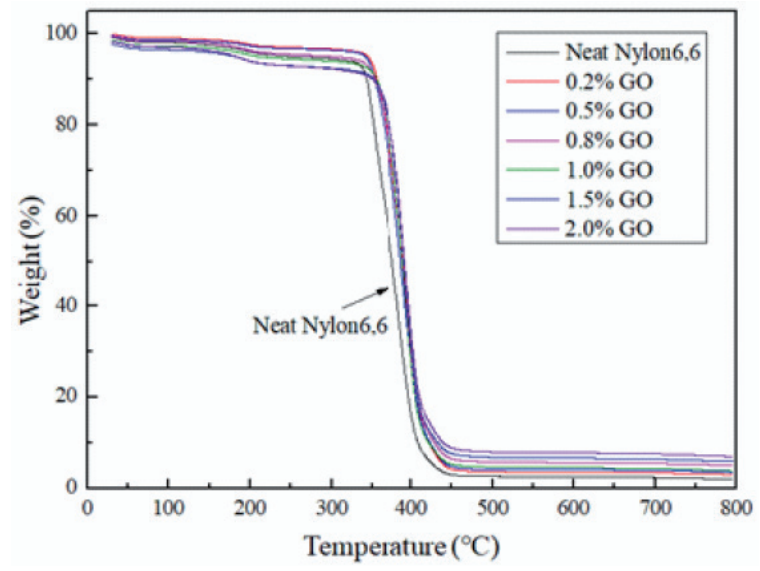

Fig. 7. (Color online) Results of TGA analysis of fiber membranes with different GO contents.

Table 3

Thermal data of composite fiber membranes.

\begin{tabular}{llc}
\hline Sample & $T_{d}\left({ }^{\circ} \mathrm{C}\right)$ & $\Delta T\left({ }^{\circ} \mathrm{C}\right)$ \\
\hline Nylon 6,6 & 352.89 & 0 \\
Nylon 6,6/0.2\%GO & 389.42 & 36.53 \\
Nylon 6,6/0.5\%GO & 390.07 & 37.18 \\
Nylon 6,6/0.8\%GO & 391.69 & 38.8 \\
Nylon 6,6/1.0\%GO & 393.31 & 40.42 \\
Nylon 6,6/1.5\%GO & 393.53 & 40.64 \\
Nylon 6,6/2.0\%GO & 394.1 & 41.21 \\
\hline
\end{tabular}


Fig. 8 shows the results of the derivative thermogravimetry (DTG) analysis of composite fiber membranes with different GO contents. The amount of fiber membranes increased and the thermal properties stabilized with increasing amount of GO added.

\subsection{Air filtration performance test}

\subsubsection{Air permeability}

A simple gas flux detection device used in an experiment to evaluate the air filtration performance of different membranes is shown in Fig. 9. A floating flowmeter was placed at both the outlet and the inlet, and a fiber membrane cut to a size of $5 \times 5 \mathrm{~cm}^{2}$ was intercalated into the serum bottle. The air flow per minute was $100 \mathrm{ml}$. After the air passed through the fiber membrane, the gas flow rate was measured with the floating flowmeter installed at the outlet. The data is shown in Table 4. The results indicated that increasing the GO content increased the membrane permeability. When the GO content was increased from 0.2 to 0.8 $\mathrm{wt} \%$, the membrane permeability was increased from 72 to $80 \mathrm{ml} / \mathrm{min}$. When GO was added, the solvent could not surround the functional powder, leading to the agglomeration of fibers and a decrease in air flux.

\subsubsection{Air particle test}

Incense was burned in a serum bottle and a pump blew the smoke-containing air through the membrane (Fig. 9). After air passed through the membrane, we used a handheld microparticle counter to detect suspended particles in the air. As shown in Table 4, the original membrane removed $78.0 \%$ of PM10 and 79.6\% of PM2.5. With increasing GO content, the filtration efficiency of the membrane increased. When $0.8 \mathrm{wt} \% \mathrm{GO}$ was added, the membrane filtration efficiency for PM10 and PM2.5 was more than 98\%. This is because of the micropores formed by the thin fibers of the Nylon 6,6/GO composite membranes. These micropores intercepted the

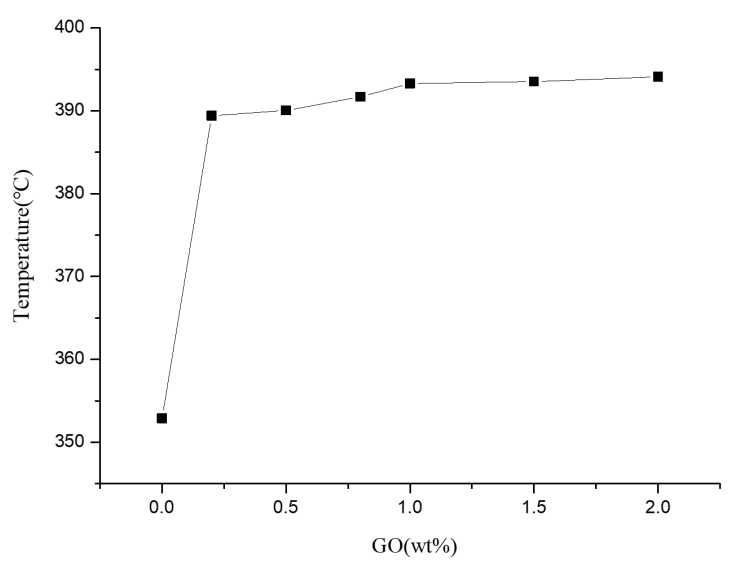

Fig. 8. Results of DTG analysis of composite fiber membranes with different GO contents.

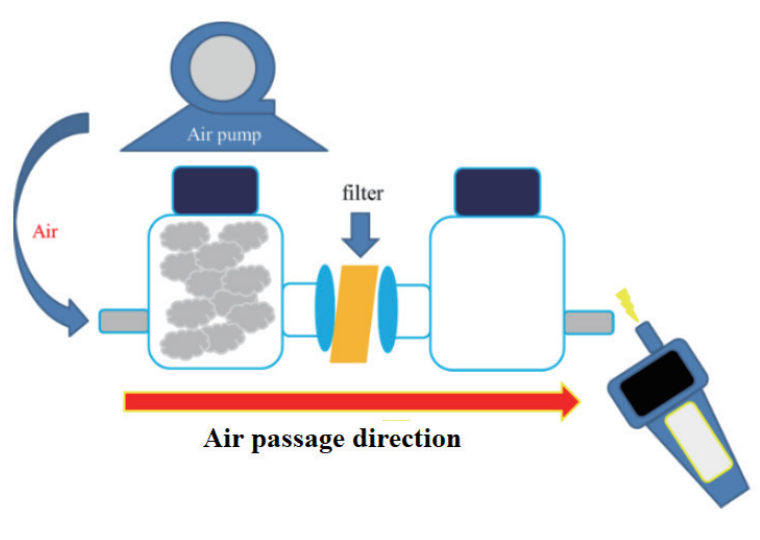

Fig. 9. (Color online) Simple gas flux detection device. 
Table 4

Filtration particle data of composite fiber membranes (incense filter).

\begin{tabular}{lcrcc}
\hline $\begin{array}{l}\text { Fiber } \\
\text { membrane }\end{array}$ & $\begin{array}{c}\text { Particle size } \\
(\mu \mathrm{m})\end{array}$ & $\begin{array}{c}\text { Particle quantity } \\
\text { (before filtration) }\end{array}$ & $\begin{array}{c}\text { Particle quantity } \\
\text { (after filtration) }\end{array}$ & $\begin{array}{c}\text { Removal } \\
(\%)\end{array}$ \\
\hline \multirow{3}{*}{ Nylon } & 10 & 15961 & 3520 & 78.0 \\
& 2.5 & 224409 & 45817 & 79.6 \\
& 1 & 3737394 & 1548245 & 58.6 \\
\hline \multirow{3}{*}{0.2} & 10 & 16485 & 2478 & 85.0 \\
& 2.5 & 231564 & 34865 & 84.9 \\
& 1 & 3364684 & 13359846 & 59.6 \\
0.5 & 10 & 16325 & 1758 & 89.3 \\
& 2.5 & 220998 & 24153 & 89.1 \\
& 1 & 36484651 & 1268495 & 65.2 \\
\hline \multirow{4}{*}{0.8} & 10 & 16219 & 245 & 98.6 \\
& 2.5 & 230608 & 2841 & 98.8 \\
& 1 & 3734634 & 946857 & 75.4 \\
\hline \multirow{3}{*}{1.0} & 10 & 15198 & 269 & 98.3 \\
& 2.5 & 240513 & 3085 & 98.6 \\
& 1 & 1524684 & 852568 & 77.1 \\
\hline \multirow{3}{*}{1.5} & 10 & 15985 & 457 & 97.0 \\
& 2.5 & 245629 & 2816 & 98.8 \\
& 1 & 943215 & 359816 & 76.4 \\
\hline & 10 & 15985 & 526 & 97.3 \\
& 2.5 & 245629 & 2978 & 97.6 \\
& 1 & 946251 & 285987 & 69.8 \\
\hline & & & &
\end{tabular}

suspended particles in the air. However, the PM1 particle size is too small for the micropores to intercept the particles. Thus, the microparticles can be adsorbed by the electrostatic force and fewer of them can be intercepted. Thus, the filtration efficiency of PM1 by the Nylon 6,6/GO composite membranes was about $75.4 \%$.

\subsubsection{Morphology of composite fiber membranes}

In Figs. 10(a)-10(c), we can see the suspended particles intercepted by the fiber surface. These three figures show that the suspended particles can indeed be intercepted by the fibers and that the fibers are not destroyed by the high temperature or chemical compounds generated by the burning incense. It can be seen in Fig. 10 that the smallest suspended particles are adsorbed on the fiber surface by the electrostatic force.

\subsubsection{Elemental analysis of composite fiber membranes}

Burning incense produces many sulfur and nitrogen oxides. We used energy dispersive spectrum (EDS) analysis to analyze the elements in the materials present on the fibers. The results in Fig. 11 clearly show the presence of sulfur, nitrogen, and oxygen. This proves that the fiber membranes prepared in this study successfully intercepted the pollutants. 


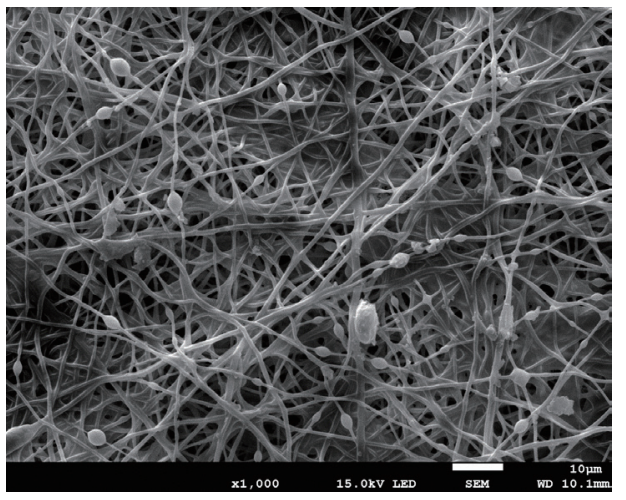

(a)

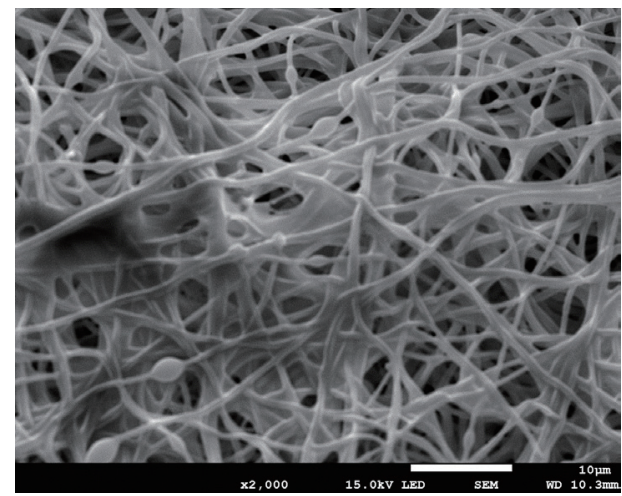

(b)

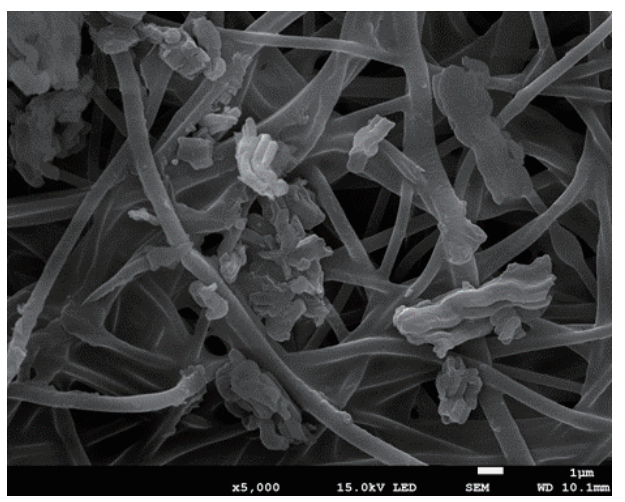

(c)

Fig. 10. SEM images of fibers after filtration.
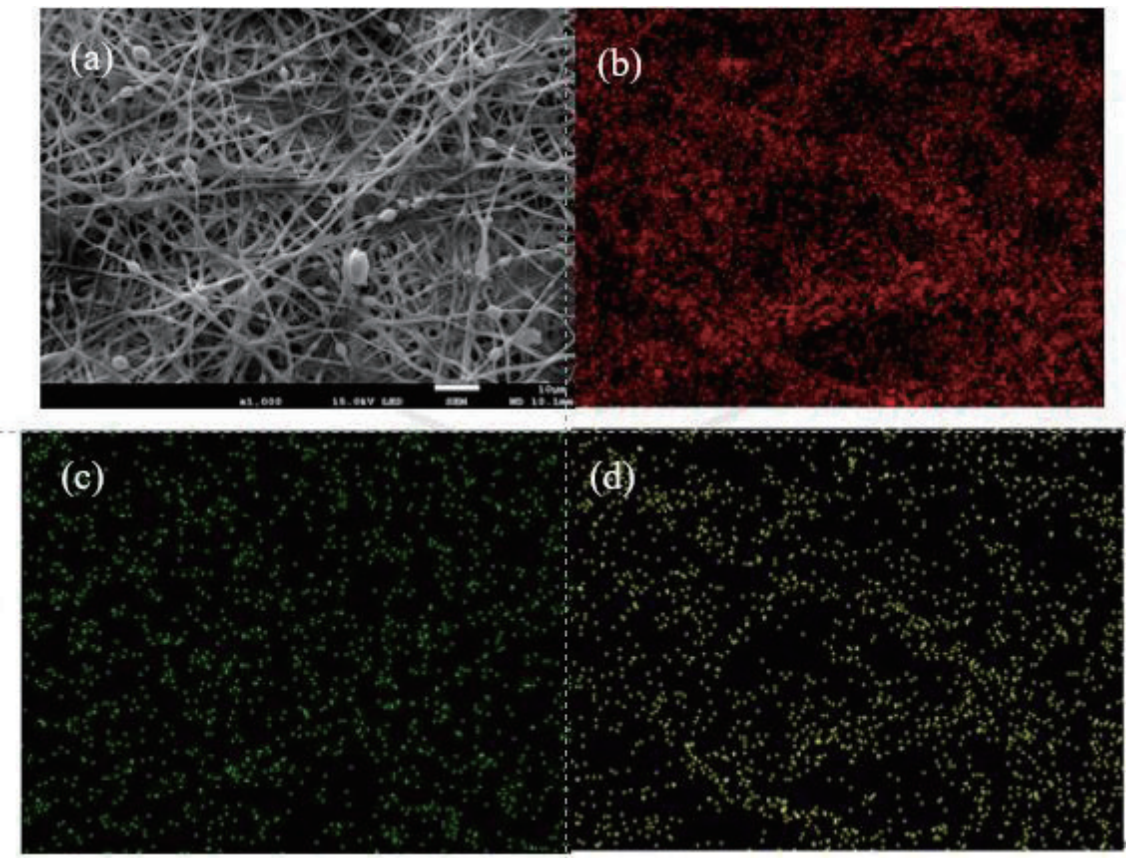

Fig. 11. (Color online) (a) SEM image and results of elemental analysis after filtration for (b) oxygen, (c) sulfur, and (d) nitrogen. 


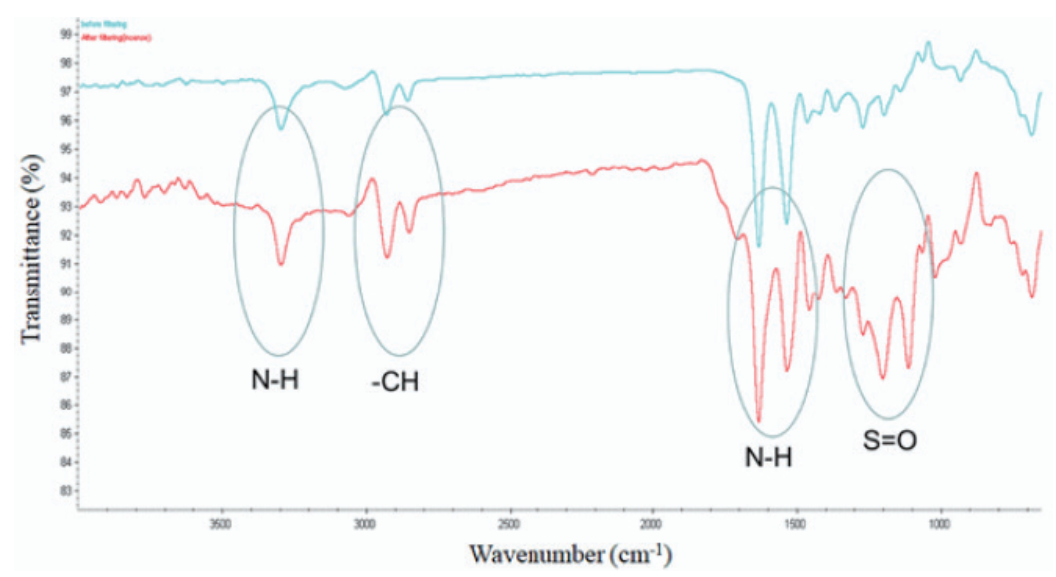

Fig. 12. (Color online) FTIR-ATR spectra of the fiber membrane after the filtration.

\subsubsection{FTIR-ATR spectra}

Figure 12 shows the FTIR-ATR spectra of a filter membrane before and after filtration. The two peaks at $1100-1200 \mathrm{~cm}^{-1}$ are $\mathrm{S}=\mathrm{O}$ tensile vibrations, the peak at about $1600-1700 \mathrm{~cm}^{-1}$ is the N-H bending vibration, that at $3200-3500 \mathrm{~cm}^{-1}$ is the N-H tensile vibration, and that at $2800-3000 \mathrm{~cm}^{-1}$ is the $-\mathrm{CH}$ tensile vibration. These characteristic peaks prove the effective filtration of the membrane.

\section{Conclusions}

In this study, we prepared GO by Hummer's method, then identified the GO structure by TEM, SEM, and Raman spectroscopy. It was found that the number of GO layers was 4-10 and the ID/IG value was 1.0647. These findings proved that the surface structure of the material was improved owing to the grafting and defects of the oxygen-containing groups. Moreover, we prepared Nylon 6,6/GO composite fiber membranes by electrospinning. The results show that the use of a solvent ratio of 3:2 (TFA:acetone) to prepare the membrane resulted in the highest stress of the membrane. Because the solvent caused the formation of hydrogen bonds between the polymer chains in the solution, the mechanical strength was greatly increased. When the amount of GO added was increased, thin fibers were formed, decreasing the fiber porosity and enhancing the possibility of intercepting minute particles. When $0.8 \mathrm{wt} \% \mathrm{GO}$ was added, the filtration efficiency of PM2.5 was $98.8 \%$ and the air flux of the fiber membrane was $78 \mathrm{ml} / \mathrm{min}$. From these results, it was found that the prepared fiber membrane had good filtration efficiency and stability, and is expected to be used in filtration supplies, masks, and so forth.

\section{References}

1 C. V. Boys: Proc. Phys. Soc. 9 (1887). https://doi.org/10.1088/1478-7814/9/1/303

2 J. F. Cooley: GB Patent (1900) 06385.

3 J. F. Cooley: US Patent (1902) 692, 631.

4 W. J. Morton: US Patent (1900) 705, 691. 
5 A. Gupta, G. Chen, P. Joshi, S. Tadigadapa, and P. C. Eklund: Nano Lett. 6 (2006) 2667.

6 E. L. Papadopoulou, F. Pignatelli, S. Marras, L. Marini, A. Davis, A. Athanassiou, and I. S. Bayer: RSC Adv. 6 (2016) 6823.

7 H. R. Pant, M. P. Bajgai, K. T. Nam, K. H. Chu, S.-J. Park, and H. Y. Kim: Mater. Lett. 64 (2010) 2087. 